\title{
LOOP SPACE HOMOLOGY OF SPACES OF SMALL CATEGORY
}

\author{
YVES FÉLIX AND JEAN-CLAUDE THOMAS
}

\begin{abstract}
Only little is known concerning $H_{*}(\Omega X ; \mathbf{k})$, the loop space homology of a finite CW complex $X$ with coefficients in a field $\mathbf{k}$. A space $X$ is called an $r$-cone if there exists a filtration $*=X_{0} \subset X_{1} \subset \cdots \subset X_{r}=X$, such that $X_{i}$ has the homotopy type of the cofibre of a map from a wedge of sphere into $X_{i-1}$. Denote by $A_{X}$ the sub-Hopf algebra image of $H_{*}\left(\Omega X_{1}\right)$. We prove then that for a graded $r$-cone, $r \leq 3$, there exists an isomorphism $A_{X} \otimes T(U) \stackrel{\cong}{\rightrightarrows} H_{*}(\Omega X)$.
\end{abstract}

\section{INTRODUCTION}

The structure of $H_{*}(\Omega X ; \mathbf{k})$, the loop space homology of a finite CW complex $X$ with coefficients in a field $\mathbf{k}$, is an exciting and interesting subject. In particular it has been conjectured that either the growth of $H_{*}(\Omega X ; \mathbf{k})$ is polynomial or else that $H_{*}(\Omega X ; \mathbf{k})$ contains a free Lie algebra on two generators. On the other hand some important results concerning the depth of $H_{*}(\Omega X ; \mathbf{k})$ have been recently obtained by Halperin, Lemaire, and the authors [8].

In this note we precise the structure of the Pontrjagin algebra, $H_{*}(\Omega X ; \mathbf{k})$, when $X$ is an $r$-cone with $r$ small.

Recall that an $r$-cone, $r \geq 0$ (resp. a finite $r$-cone) $X$ is a sequence

$$
*=X_{0} \subset X_{1} \subset \cdots \subset X_{r}=X \text {, }
$$

where $X_{i}, i \geq 1$, is the cofiber of a map $g_{i}, W_{i} \stackrel{g_{i}}{\rightarrow} X_{i-1} \stackrel{f_{i}}{\rightarrow} X_{i}$, with $W_{i}$ a wedge of spheres (resp. a wedge of finitely many spheres) and $\left(f_{i}\right)_{*}: \widetilde{H}_{*}\left(X_{i-1} ; \mathbf{k}\right)$ $\rightarrow \widetilde{H}_{*}\left(X_{i} ; \mathbf{k}\right)$ is trivial.

The image $A_{X}$ of $H_{*}\left(\Omega X_{1} ; \mathbf{k}\right)$ into $H_{*}(\Omega X ; \mathbf{k})$ is a graded sub-Hopf algebra of $H_{*}(\Omega X ; \mathbf{k})$, isomorphic to the quotient Hopf algebra $H_{*}\left(\Omega X_{1} ; R\right) / \operatorname{Ker}\left(\Omega f_{2}\right)_{*}$ when $X$ is a 2 -cone.

An $r$-cone is a space of Lusternik-Schnirelmann category (LS-category) less than or equal to $r$.

A space $X$ of LS-category one is a co- $H$-space. The Bott-Samelson theorem shows that $H_{*}(\Omega X ; \mathbf{k})$ is then a tensor algebra, $T(V)$, with

$$
V=\bigoplus_{i \geq 0} V_{i} \text { and } V_{i}=\widetilde{H}_{i+1}(X ; \mathbf{k}) \text {. }
$$

Received by the editors May 6, 1991.

1991 Mathematics Subject Classification. Primary 57P35, 57P10, 57T25, 55P62.

Key words and phrases. Loop space, r-cones, Lusternik-Schnirelmann category, Hopf algebra, global dimension, depth.

This research partially supported by a CNRS-CGRI travel grant held by the authors. 
In the study of the loop space homology of spaces, the first nontrivial case is given by the 1-connected $\mathrm{CW}$-complex of finite type of category 2 . In this case:

(a) the numbers $\sum_{i=0}^{n} \operatorname{dim} H_{i}(\Omega X ; \mathbf{k})$ grow either polynomially or else the algebra $H_{*}(\Omega X ; \mathbf{k})$ contains a tensor algebra on two generators [9].

(b) When $\mathbf{k}=\mathbb{Q}$, there exists a short exact sequence of graded Lie algebras,

$$
0 \rightarrow \mathbb{L}(U) \rightarrow \pi_{*}(\Omega X) \otimes \mathbb{Q} \rightarrow L_{X} \rightarrow 0,
$$

with $A_{X}=\mathbf{U} L_{X}[11]$.

We will precise (a) (Theorem 1), and generalize (b) for some spaces of LScategory less or equal to 4 (Theorems 2 and 3 ).

In order to make our results more precise, we have to introduce what we shall call algebraic $r$-cones.

Recall that a free model for an augmented chain $R$-algebra $A$ is a chain algebra map $\varphi:(T(V), d) \rightarrow A$ such that

(i) $V=\bigoplus_{i \geq 0} V_{i}$

(ii) $\varphi$ induces an isomorphism $\varphi_{*}: H(T(V), d) \rightarrow H(A)$.

When $X$ is a 1-connected CW-complex, Adams and Hilton [1] have already established the existence of a model of the chain algebra, $A=C_{*}(\Omega X ; \mathbf{k})$, consisting of the singular cubical chains modulo degeneracies. As shown in [12, Appendix], one can choose $V$ such that

$$
V_{i} \cong \widetilde{H}_{i-1}(X ; \mathbf{k}), \quad i \geq 2 \text {. }
$$

In this case the chain algebra $(T(V), d)$ is called minimal.

The same inductive construction as in [1], following the cone filtration instead of the skeleton filtration gives a filtered fiee model for a 1 -connected $r$-cone $X$. In this case, $V$ is a bigraded vector space satisfying

(a) $V=\bigoplus_{0 \leq i \leq r-1,0 \leq j} V_{i, j}, d\left(V_{i, j}\right) \subset \bigoplus_{s<i, s+t=i+j-1}(T(V))_{s, t}$,

(b) $H_{*}\left(\Omega X_{i} ; \mathbf{k}\right)=H\left(T\left(V_{\leq i}\right), d\right)$ for $i \geq 0$,

(c) $A_{X}=T(V) /\left(d(V)+V_{+, *}\right)$ is a sub-Hopf algebra of the graded connected cocommutative Hopf algebra $H(T(V), d)$.

By definition, a space $X$ such that $C_{*}(\Omega X ; \mathbf{k})$ admits a free model, $(T(V), d)$, satisfying the conditions (a) and (c) is called an algebraic r-cone over $\mathbf{k}$ with associated model $(T(V), d)$.

If, moreover each $V_{i}$ is a graded vector space of finite type (resp. if $\operatorname{dim} V_{i}<$ $\infty$ for any $i$ ) we shall say that $X$ is an algebraic $r$-cone of finite type (resp. a finite algebraic $r$-cone).

Clearly, any $r$-cone is an algebraic $r$-cone over any field $\mathbf{k}$. The converse is false since, for instance for $r=1$, there exists co- $H$-spaces which are not wedges of spheres.

Among the class of algebraic $r$-cones we distinguish those satisfying the strengthened condition:

$\left(\mathbf{a}^{\prime}\right) d\left(V_{i, j}\right) \subset(T(V))_{i-1, j}$.

These algebraic $r$-cones will be called graded $r$-cones. Clearly an algebraic 2-cone is always a graded 2-cone.

For a graded $r$-cone, $X$, over $\mathbf{k}$, the homology of $\Omega X$ is a bigraded vector space:

$$
H_{*}(\Omega X ; \mathbf{k})=\bigoplus H_{i, j}(\Omega X ; \mathbf{k}) .
$$


The integer $i$ is called the filtered degree and $i+j$ the total degree. Later on, we shall simply denote $H_{i, *}(\Omega X ; \mathbf{k})$ by $H_{i}$. In particular $H_{0}=A_{X}$. We can now state

Theorem 1. If $X$ is a 1-connected algebraic 2-cone of finite type over a field $\mathbf{k}$, then there exists a graded subspace $U$ of $H_{1}$ such that

(a) $T(U)$ is a subalgebra of $H_{*}(\Omega X ; \mathbf{k})$,

(b) The multiplication law induces two linear isomorphisms

$$
T(U) \otimes A_{X} \cong H_{*}(\Omega X ; \mathbf{k}) \stackrel{\cong}{\leftrightarrows} A_{X} \otimes T(U),
$$

(c) If $\operatorname{dim} H_{*}(X ; \mathbf{k}) \geq 3$ then either $U=0$ or else $\operatorname{dim} U \geq 2$.

Notice that $U=0$ if and only if there is an isomorphism of Hopf algebras

$$
H_{*}(\Omega X ; \mathbf{k}) \stackrel{\cong}{\rightrightarrows} H_{*}\left(\Omega X_{0} ; \mathbf{k}\right) / \operatorname{Ker}(\Omega f)_{*}=A_{X} .
$$

In this case the attaching map $g_{2}$ is called $\mathbf{k}$-inert [12] or strongly free [3].

Example 1. Let $X$ be the 6-skeleton of the product $S_{a}^{3} \times S_{b}^{3} \times S_{c}^{3}$, i.e., the fat wedge of the three spheres $S_{a}^{3}, S_{b}^{3}, S_{c}^{3}$. Then $H_{*}(\Omega X ; \mathbf{k}) \cong A_{X} \otimes T(U)$; $A_{X}=\mathbf{k}[a, b, c]$, with $\operatorname{deg}(a)=\operatorname{deg}(b)=\operatorname{deg}(c)=2$ and $U=\alpha \cdot \mathbf{k} \otimes A_{X}$, with $\operatorname{deg}(\alpha)=7$.

Example 2. The restriction of the suspension homomorphism

$$
\sigma_{*}: H_{*}(\Omega X ; \mathbf{k}) \rightarrow H_{*-1}(X ; \mathbf{k})
$$

to $V_{0}$ is an injective map. Denote by $K_{\sigma}$ the kernel of the linear homomorphism induced by $\sigma_{*}$ on the vector space of indecomposable elements of $H_{*}(\Omega X)$ :

$$
0 \rightarrow K_{\sigma} \rightarrow Q\left(H_{*}(\Omega X)\right) \stackrel{\sigma}{\rightarrow} H_{*-1}(X) .
$$

This yields the following corollary.

Corollary. The subalgebra of $H_{*}(\Omega X ; \mathbf{k})$ generated by $K_{\sigma}$ is free: $T\left(K_{\sigma}\right)$ injects into $H_{*}(\Omega X)$.

Theorem 2. If $X$ is a 1-connected finite graded 3-cone over a field $\mathbf{k}$ with associated free model $(T(V), d)$, then there exist $U_{1}$ and $U_{2}$ respectively subspaces of $H_{1}$ and $H_{2}$ such that $T\left(U_{1} \oplus U_{2}\right)$ is a free subalgebra of $H_{*}(\Omega X ; R)$ and such that the multiplication law induces two linear isomorphisms

$$
A_{X} \otimes T\left(U_{1} \oplus U_{2}\right) \cong H_{*}(\Omega X ; \mathbf{k}) \cong T\left(U_{1} \oplus U_{2}\right) \otimes A_{X} .
$$

Over $\mathbb{Q}$, we have

$$
H_{*}(T(V), d) \cong H_{*}(\Omega X ; \mathbb{Q}) \cong \mathbf{U}\left(\pi_{*}(\Omega X \otimes \mathbb{Q})\right) \text { and } A_{X}=\mathbf{U} L_{X} .
$$

In particular, the rational homotopy Lie algebra of a graded 3-cone appears as an extension of $L_{X}$ by a free graded Lie algebra,

$$
0 \rightarrow \mathbb{L}\left(U_{1} \oplus U_{2}\right) \rightarrow \pi_{*}(\Omega X) \otimes \mathbb{Q} \rightarrow L_{X} \rightarrow 0 .
$$

Theorem 3. If $X$ is a 1-connected finite graded 4-cone over $\mathbb{Q}$, then the structure of $\pi_{*}(\Omega X) \otimes \mathbb{Q}$ is given by the following two short exact sequences:

$$
\begin{gathered}
0 \rightarrow P \rightarrow \pi_{*}(\Omega X) \otimes \mathbb{Q} \rightarrow L_{X} \rightarrow 0, \\
0 \rightarrow \mathbb{L}(U) \rightarrow P \rightarrow N \rightarrow 0,
\end{gathered}
$$


where $N$ is the quotient of a free Lie algebra by an ideal generated by quadratic elements. Moreover, if $X$ is a 4-cone the generators of $N$ are in the image of the canonical map $H_{*}\left(\Omega X_{2} ; \mathbb{Q}\right) \rightarrow H_{*}\left(\Omega X_{4} ; \mathbb{Q}\right)=H_{*}(\Omega X ; \mathbb{Q})$.

To prove these theorems, we use the $I$-adic spectral sequence [5]. For the convenience of the reader some of its properties are recalled in $\S 2$. In the first section we prove that algebraic $r$-cones $X$ satisfy M-cat $(X) \leq r$ (see below for the definition). The other sections are devoted to the proofs of Theorems 1 to 3.

\section{M-CATEGORY OF AN $r$-CONE}

It is well known that the LS-category of an $r$-cone is less or equal to $r$. In this chapter we establish an analogous result for algebraic $r$-cones.

In [12], Halperin and Lemaire have introduced a new and very powerful approximation of the category. They called it M-cat. We first recall the definition of M-cat $X$ for a simply connected space $X$.

Consider a free model $(T(W), d)$ of $C^{*}(X ; \mathbf{k})$ in the category of cochain algebras. Any cochain algebra map $(T(W), d) \rightarrow\left(A, d_{A}\right)$ factors as $(T(W), d)$ $\rightarrow(T(W \oplus U), d) \stackrel{\cong}{\rightrightarrows}\left(A, d_{A}\right)$. In particular, for any $m$ we can factor the quotient map

$$
\rho_{m}:(T(W), d) \rightarrow\left(T(W) / T^{>m}(W), \bar{d}\right)
$$

as

$$
(T(W), d) \stackrel{j}{\rightarrow}\left(T\left(W \oplus U_{m}\right), d\right) \stackrel{\cong}{\rightrightarrows}\left(T(W) / T^{>m}(W), \bar{d}\right) .
$$

Definition. $\mathrm{M}-\operatorname{cat}(X)=\operatorname{M-cat}((T(V), d))$ is the least integer $m$ such that there exists a morphism

$$
r:\left(T\left(W \oplus U_{m}\right), d\right) \rightarrow(T(W), d)
$$

of left $(T(V), d)$ differential modules such that $r j=\operatorname{Id}_{T(V)}$.

The M-category is an approximation of the category in the sense that [12] $\operatorname{M-cat}(X) \leq \operatorname{cat}(X)$. We now consider a graded algebra,

$$
R=\mathbf{k} \oplus \bigoplus_{k \geq 1} R^{k}
$$

The global dimension of $R$ (possibly $\infty$ ) is the largest integer $n$ such that $\operatorname{Ext}_{R}^{n}(\mathbf{k}, \mathbf{k}) \neq 0$. The depth of $R$ (possibly $\infty$ ) is the least integer $n$ such that $\operatorname{Ext}_{R}^{n}(\mathbf{k}, R) \neq 0$.

Now, by $\left[8\right.$, Theorem $\left.\mathrm{A}^{\prime}\right]$ we have

Proposition 1.1. Let $X$ be a simply connected space, $\mathbf{k}$ a field, and assume that each $H_{i}(X ; \mathbf{k})$ is finite dimensional. Then

(1) $\operatorname{depth} H_{*}(\Omega X ; \mathbf{k}) \leq \mathrm{M}$-cat $X \leq \mathrm{gl} \operatorname{dim} H_{*}(\Omega X ; \mathbf{k})$.

(2) If depth $H_{*}(\Omega X ; \mathbf{k})=\mathrm{M}$-cat $X$ then

$$
\operatorname{depth} H_{*}(\Omega X ; \mathbf{k})=\operatorname{gldim} H_{*}(\Omega X ; \mathbf{k}) .
$$

Here we prove 
Proposition 1.2. Let $X$ be a simply connected space, $\mathbf{k}$ a field, and assume that each $H_{i}(X ; \mathbf{k})$ is finite dimensional. If $X$ is an algebraic $n$-cone then $\mathrm{M}-\operatorname{cat}(X) \leq n$.

Proof of Proposition 1.2. Let $(T(V), d) \rightarrow C_{*}(\Omega X ; \mathbf{k})$ be a free model associated to the structure of an algebraic $n$-cone. For every graded vector space $E$, we will denote by $E^{\vee}$ the graded dual of $E$. The letter $\underline{B}$ will denote the (reduced) bar construction. We then have the following quasi-isomorphisms of differential graded coalgebras:

$$
\underline{B}(T(V), d) \rightarrow \underline{B}\left(C_{*}(\Omega X ; \mathbf{k})\right) \leftarrow C_{*}(X ; \mathbf{k}) .
$$

Write $\left(T(W), d^{\vee}\right)=(\underline{B}(T(V), d))^{\vee}$ the dual cochain algebra. By definition of $\underline{B}, W^{i, j} \cong\left(T^{+}(V)\right)_{i-1, j}$ and $d^{\vee}$ is a differential of bidegree $(1,0)$.

Let $M=\mathbf{k} \oplus T^{n+1}(W)$, then $M^{i, *}=0$ for $0<i \leq n$. On the other hand, by [8, proof of Theorem $\mathrm{A}^{\prime}$ ], we have a differential $D$ and a quasi-isomorphism of $\left(T(W), d^{\vee}\right)$-differential modules:

$$
(T(W) \otimes M, D) \stackrel{\varphi}{\rightarrow}\left(T(W) / T^{>n}(W), \overline{d^{\vee}}\right) .
$$

$\mathrm{M}$-cat $(X) \leq n$ if and only if there exists a morphism

$$
r:(T(W) \otimes M, D) \rightarrow\left(T(W), d^{\vee}\right)
$$

of differential $\left(T(W), d^{\vee}\right)$-modules such that the composite

$$
\left(T(W), d^{\vee}\right) \longmapsto(T(W) \otimes M, D) \stackrel{r}{\rightarrow}\left(T(W), d^{\vee}\right)
$$

equals $\operatorname{Id}_{T(V)}$.

By [10] we know that the composite $q$ :

$$
\begin{aligned}
\underline{B}(T(V), d)^{\vee} & =\left(T(W), d^{\vee}\right) \rightarrow\left(T(W) / T^{>2}(W), d^{\vee}\right) \\
& =\left(\mathbf{k} \oplus W, d^{\prime}\right) \rightarrow\left(\mathbf{k} \oplus s V, s d_{V}\right)^{\vee}
\end{aligned}
$$

is a quasi-isomorphism of bigraded differential vector spaces. Thus

$$
H^{i, *}\left(T(W), d^{\vee}\right)=0
$$

for $i>n$ and any cocycle in $T^{n+1}(W)$ is a coboundary. This makes possible the construction of a retraction $r$.

\section{THE I-ADIC SPECTRAL SEQUENCE}

The $I$-adic spectral sequence is defined in [5]. For the convenience of the reader, we recall here its construction and main properties.

Let $(T(V), d)$ be a chain algebra over a field $\mathbf{k}$ such that $V=\bigoplus_{i \geq 0} V_{i}$ is a graded vector space satisfying $d\left(V_{i}\right) \subset T(V)_{i-1}$. We denote by $I$ the kernel of the composite $T(V) \rightarrow T\left(V_{0}\right) \rightarrow H_{0}$. This gives a short exact sequence of graded algebras: $I \rightarrow T(V) \rightarrow H_{0}$. It appears that $I$ is a free left (and right) $T(V)$-module: $I=T(V) \otimes M$ for some subspace $M$ of $I$.

The powers of the ideal $I$ define an increasing filtration

$$
F_{0}=T(V), \quad F_{-p}=I^{p}, \quad p>0,
$$

which generates a second quadrant spectral sequence of $H_{0}$-algebras satisfying:

(1) $E_{-p, q}^{0}=\left(I^{p} / I^{p+1}\right)_{-p+q} \cong\left(H_{0} \otimes\left(\otimes^{p} M\right)\right)_{-p+q}$. 
(2) The multiplication law induces an isomorphism of $H_{0}$-modules $\otimes_{H_{0}}^{p}\left(I / I^{2}\right) \rightarrow\left(I^{p} / I^{p+1}\right)=E_{p, *}^{0}$ and $\left(I / I^{2}\right)_{n} \cong H_{0} \otimes V_{n} \otimes H_{0}$ for any $n>0$.

(3) $E_{-p, q}^{1}=0$ if $q<2 p$, therefore the $I$-adic spectral sequence converges.

(4) If $V=V_{\leq n}$, then $E_{-p, q}^{0}=0$ for $q>p \cdot(n+1)$.

(5) If $H_{r}(T(V), d)=0$ for $0<r<m$, then $E_{-p, q}^{1}=0$ for $q<r \cdot(m+1)$.

Remark that property (3) gives for $n \leq 2 p$ the short exact sequences

$$
E_{-p, n}^{0} \cap \operatorname{ker} d_{0} \longmapsto E_{-p, n}^{0} \stackrel{d_{0}}{\rightarrow} E_{-p, n-1}^{0} \cap \operatorname{ker} d_{0} .
$$

As $I^{n} / I^{n+1}$ is a free $H_{0}$-module, $E_{-n, 0}^{0}=E_{-n, 0}^{0} \cap \operatorname{ker} d_{0}$ is a projective $H_{0^{-}}$ module. An induction on $n$ shows then that, for each $n \leq 2 p, E_{-p, n}^{0} \cap \operatorname{ker} d_{0}$ is a projective $H_{0}$-module.

Lemma 2.1. Let $(T(V), d)$ be a chain algebra over a field $\mathbf{k}$ such that $V=V_{0} \oplus$ $V_{1}$ is a vector space, $d\left(V_{i}\right) \subset T(V)_{i-1}$. Then there exists a natural isomorphism of algebras $T_{H_{0}}\left(H_{1}(T(V))\right) \rightarrow E_{*, *}^{1}$.

Proof of Lemma 2.1. By properties (3) and (4) $E_{p, q}^{1}=0$ if $q \neq 2 p$. Therefore the $I$-adic spectral sequence collapses at the $E^{1}$ level and

$$
H_{p}(T(V), d)=E_{-p, 2 p}^{1} .
$$

Now, $E_{-1,2}^{1}=E_{-1,2}^{0} \cap \operatorname{ker} d_{0}$ is a projective $H_{0}$-module.

On the other hand, the natural inclusion $E_{-1,2}^{1} \rightarrow E_{-1,2}^{0}$ extends to a homomorphism of differential $R$-algebras

$$
\left(T_{H_{0}}\left(E_{-1,2}^{1}\right), 0\right) \rightarrow\left(T_{H_{0}}\left(E_{-1,2}^{0}\right), d_{0}\right) \cong\left(E_{*, *}^{0}, d_{0}\right) .
$$

Thus, an induction on the tensor powers and the Künneth spectral sequence show that this homomorphism induces an isomorphism $T_{H_{0}}\left(E_{-1,2}^{1}\right) \stackrel{\cong}{\rightrightarrows} E_{*, *}^{1}$ and thus an isomorphism of algebras

$$
T_{H_{0}}\left(H_{1}(T(V))\right) \stackrel{\cong}{\rightrightarrows} E_{*, *}^{1} \text {. }
$$

\section{Proof of Theorem 1}

Consider a minimal free model associated to an algebraic 2-cone $X$. We thus have

(i) $V=V_{0} \oplus V_{1}, d\left(V_{0}\right)=0$,

(ii) $V_{0}=\bigoplus_{1 \leq i \leq g} V_{0, i}, V_{1}=\bigoplus_{1 \leq j \leq r} V_{1, j-1}$,

(iii) $d\left(V_{0}\right)=\overline{0}, d\left(V_{1, j}\right) \subset(T(V))_{0, j}$,

(iv) $H_{0}(T(V), d) \cong A_{X}$.

The first two parts of Theorem 2 are now easy consequences of Lemma 2.1.

Indeed,

$$
H_{*}(\Omega X ; \mathbf{k}) \cong T_{H_{0}}\left(H_{1}(T(V))\right),
$$

and since $H_{1}(T(V))$ is a free left and right $H_{0}$-module [14, Lemma 5], we put $H_{1}(T(V))=U \otimes A_{X}$ or $A_{X} \otimes U$.

The last part of Theorem 1 requires some preliminaries: 
Lemma 3.1. If $A_{X}$ is an algebra of global dimension 2 then $A_{X}=H(T(V), d)$. Proof of Lemma 3.1. Any graded connected chain algebra with trivial differential, $(B, 0)$ admits a free bigraded model [12], $f:(T(Z), d) \stackrel{\cong}{\rightrightarrows}(B, 0)$ which is a bigraded differential chain algebra satisfying:

(1) $Z=\bigoplus_{p \geq 0, q \geq 0} Z_{p, q}$.

(2) $d\left(Z_{p, q}\right) \subset T^{\geq 2}(Z)_{p-1, q}$

(3) $H_{0, *}(T(Z), d) \cong B, H_{i, *}(T(Z), d)=0, i>0$.

(4) $(T(Z), d)$ is unique up to an isomorphism of bigraded differential algebras.

Following Adams and Hilton [1], we can associate to $(T(Z), d)$ an acyclic $(T(Z), d)$-module $C=T(Z) \otimes(\mathbf{k} \oplus s Z)$ with a differential $D$ extending $d$ and defined by

(1) $D(s z)=z-s(d z), \quad z \in Z$,

(2) $s(s z)=0, z \in Z, s(x y)=(-1)^{\operatorname{deg}(x)} x s(y), x, y \in T(Z)$.

The chain complex

$$
B \otimes(\mathbf{k} \oplus s Z)=B \otimes_{T(Z)} C
$$

is then a resolution of the field $\mathbf{k}$ by free $B$-modules:

$$
\cdots B \otimes s\left(Z_{2}\right) \stackrel{d_{2}}{\rightarrow} B \otimes s\left(Z_{1}\right) \stackrel{d_{1}}{\rightarrow} B \otimes s\left(Z_{0}\right) \stackrel{d_{0}}{\rightarrow} B \rightarrow \mathbf{k} .
$$

Denote by $z$ an element of $Z_{p}$. We have

$$
D(s z)=z-s(d z) .
$$

Write $d(z)=\sum_{i} \nu_{i} \beta_{i}+\sum_{j} \gamma_{j} \cdot z_{j}$, with $\beta_{i}$ a basis of $Z_{0}$, and $z_{j}$ a basis of $\bigoplus_{i \geq 1} Z_{i}$. This implies

$$
d_{p} s(z)=\sum_{j} f\left(\gamma_{j}\right) \cdot s\left(z_{j}\right), \quad f\left(\gamma_{i}\right) \in B_{+} .
$$

Thus, $\operatorname{Tor}_{p}^{B}(\mathbf{k}, \mathbf{k})=s Z_{p-1}$.

Since the global dimension of $B$ is less or equal to 2 and since $d(Z) \subset$ $T \geq^{2}(Z)$, this last relation implies that $Z_{p}=0$ for $p \geq 2$.

Now, for $B=A_{X}$, the two minimal models $\left(T\left(\bar{Z}_{\leq 1}\right), d\right)$ and $(T(V), d)$ are isomorphic [12, Appendix], and therefore $H_{p}=0$ for any $p>0$.

Lemma 3.2. Let $G$ be a graded connected cocommutative Hopf algebra of finite type over a field $\mathbf{k}$. Then the depth of $G$ is zero if and only if $\operatorname{dim} G<\infty$.

Proof of Lemma 3.2. As

$$
\operatorname{Ext}_{G}^{0}(\mathbf{k} ; \mathbf{k})=\operatorname{Hom}_{G}(\mathbf{k}, G),
$$

one see that $\operatorname{depth}(G)=0$ if and only if the annihilator of $G_{+}$, Ann $G$, is nonzero.

$$
\text { Ann } G=\left\{x \in G \mid g x=0 \text { for all } g \in G_{+}\right\} .
$$

Clearly, if $\operatorname{dim} G<\infty$ then $\operatorname{depth}(G)=0$.

We now prove the converse, and we suppose $\operatorname{depth}(G)=0$.

(a) If $\mathbf{k}=\mathbb{Q}$. The Milnor-Moore theorem implies that $G=\mathbf{U} L$ for some Lie algebra $L=\bigoplus_{i \geq 0} L_{i}$. In this case $\operatorname{depth}(G)=\operatorname{dim}_{\mathbf{k}} L_{\text {even }}$ [7]. Thus, $L$ is concentrated in odd degrees and the Poincare-Birkoff-Witt theorem [14] implies that $\operatorname{dim}_{\mathbf{k}} U L<\infty$. 
(b) If $\mathbf{k}=\mathbb{Z}_{p}$ for a fixed prime $p$. Let $\bar{\Delta}: G_{+} \rightarrow G_{+} \otimes G_{+}$be the reduced diagonal. Define $\bar{\Delta}_{n}: G_{+} \rightarrow G_{+} \otimes G_{+} \otimes \cdots \otimes G_{+}(n+1$ times $)$ by

$$
\bar{\Delta}_{0}=\bar{\Delta}, \quad \bar{\Delta}_{n+1}=(\bar{\Delta} \otimes 1 \cdots 1 \otimes 1) \circ \bar{\Delta}_{n}
$$

and

$$
F^{0} G=R, \quad F^{n+1} G=\operatorname{Ker} \bar{\Delta}_{n}, \quad n \geq 0 .
$$

$\left(F^{p} G\right)$ is an increasing filtration of $G: F^{p} G \subset F^{p+1} G, \bigcup_{p \geq 0} F^{p} G=G$.

Set $A^{p}=F^{p} G / F^{p-1} G, A=\bigoplus_{p} A^{p}$. Then by $[6,1.3]$ the Hopf algebra $A$ is both commutative and cocommutative and $\xi\left(A_{+}\right)=0$ where $\xi(x)=x^{p}$.

The Borel theorem implies that the algebra $A$ is isomorphic to $\otimes A(i)$ where the $x_{i}$ are generators of $A$ and $A(i)=\mathbb{Z}_{p}\left[x_{i}\right]$ or $\mathbb{Z}_{p}\left[x_{i}\right] / x_{i}^{p}$ if $x_{i}$ has even degree and $\Lambda x_{i}$ if $x_{i}$ has odd degree or $p=2$.

Now, by our assumption, the annihilator of $A$ is nonzero which implies that $\operatorname{dim}_{\mathbf{k}} A=\operatorname{dim}_{\mathbf{k}} G$ is finite.

End of the proof of Theorem 1. We denote by $(T(V), d)$ a free model that gives to $X$ its algebraic 2-cone structure. We put $A=H_{*}(\Omega X ; \mathbf{k})=H(T(V), d)$ and recall that $A_{X}$ is a sub-Hopf algebra of $A$ which is not necessarily a normal subalgebra.

By Propositions 1.1 and 1.2, we reduce the proof to three cases.

First case. The global dimension of $A$ is 2 and $\operatorname{dim} A_{X}=\infty$.

The global dimension of $A_{X}$ is then 2 and Lemma 3.1 implies that $H_{1}=$ $H_{1}(T(V), d)=0$ and thus part (b) of Theorem 1 implies $U=0$.

Second case. Depth of $A=1$ and $\operatorname{dim} A_{X}=\infty$.

We suppose that $U$ is generated by only one element $u$ and we will construct a contradiction. As $T(u)$ is a normal subalgebra, we have isomorphisms

$$
A_{X} \otimes T(u) \cong A \cong T(u) \otimes A_{X} .
$$

This defines a linear automorphism of $A_{X}, b \rightarrow b^{u}$ satisfying $u \cdot b-b^{u} \cdot u \in\left(u^{2}\right)$ where $\left(u^{2}\right)$ denotes the two-sided ideal of $A$ generated by $u^{2}$.

On the other hand,

$$
\operatorname{Ext}_{A}^{1}(\mathbf{k}, A)=\operatorname{Hom}_{A}\left(A_{+}, A\right) / \operatorname{Hom}_{A}(A, A) \neq 0 .
$$

We denote by $\beta: A_{+} \rightarrow A$ a morphism of left $A$-modules representing a nontrivial element of $\operatorname{Ext}_{A}^{1}(\mathbf{k}, A)$, i.e., $\beta$ does not extend into an $A$-linear endomorphism of $A$.

By linearity, $u \cdot \beta(b)-b^{u} \cdot \beta(u)$ is an element of the right $A_{X}$-submodule $\left(u^{2}\right)+T^{+}(u) \otimes A_{X}$ for any $b \in\left(A_{X}\right)_{+}$. We decompose the element $\beta(u)$ as

$$
\beta(u)=b_{0}+u a, \quad b_{0} \in A_{X}, a \in A .
$$

Necessarily,

$$
b^{u} \cdot b_{0}=0 \text { for any } b \in\left(A_{X}\right)_{+} .
$$

As $\operatorname{dim} A_{X}=\infty$, Lemma 3.2 implies $b_{0}=0$. Now the relation $u b=b^{\prime} u$ associates to any $b \in A_{X}$ some element $b^{\prime} \in A$. This leads to the relations

$$
u \beta(b)=b^{\prime} \beta(u)=b^{\prime} u a=u b a .
$$

As $A$ is a free left $T(u)$-module, this gives $\beta(b)=b a$ for any $b \in A_{X}$. This yields a contradiction with the assumption of nontriviality of the cocycle $\beta$. 
Thus, $b_{0} \neq 0$. By the above relation, the element $b_{0}$ belongs to the annihilator of $\left(A_{X}\right)_{+}$and by Lemma $2, \operatorname{dim} A_{X}<\infty$.

Third case. $\operatorname{Dim} A_{X}<\infty$.

The Poincare series of a graded vector space $M=\bigoplus_{i} M_{i}$ will be denoted by $P(M)=\sum_{i} \operatorname{dim} M_{i} t^{i}$. In the same way, the Koszul-Poincare series of a bigraded vector space $N$ will be denoted by

$$
K P(N)=\sum_{i, j}(-1)^{i} \operatorname{dim} N_{i, j} t^{j-i} .
$$

From the obvious relation, $K P(T(V))=K P(H(T(V), d))$, we deduce

$$
\sum_{i}(-t)^{-i} P\left(T(V)_{i}\right)=\sum_{i}(-t)^{-i} P\left(H_{i}\right) .
$$

On the other hand, as $V=V_{0} \oplus V_{1}$, with $V_{0}=\bigoplus_{1 \leq i \leq g} v_{i} \mathbf{k}$, degree of $v_{i}=m_{i}$ and $V_{1}=\bigoplus_{1 \leq j \leq r} v_{j}^{\prime} \mathbf{k}$, degree of $v_{j}^{\prime}=n_{j}+1$, we have

$$
\sum_{r}(-t)^{r} P\left(T(V)_{r}\right)=(Q(t))^{-1}, \quad \text { with } Q(t)=1-\sum_{i \in I} t^{m_{i}}+\sum_{j \in J} t^{n_{j}} .
$$

We have also (since $u \in H_{1, s}$ and $A_{X}$ is finite dimensional):

$$
\sum_{i}(-t)^{i} P\left(H_{i}\right)=P\left(A_{X}\right) /\left(1+t^{s}\right),
$$

where $P\left(A_{X}\right)$ is a polynomial. Thus,

$$
P\left(A_{X}\right) Q(t)=\left(1+t^{s}\right) .
$$

So, as $P\left(A_{X}\right)$ has nonnegative coefficients, the relation

$$
P\left(A_{X}\right)(1) Q(1)=2, \quad Q(1)=1-g+r,
$$

implies

$$
\operatorname{dim} A_{X}=P\left(A_{X}\right)(1)=1 \text { or } 2 .
$$

If $A_{X}=\mathbf{k}$ then $X$ is an algebraic 1-cone thus the only case to consider is $A_{X}=\bigwedge x$. In this case $g=1, r=1$, and $A$ is the homology of the chain algebra $\left(T(x, y), d y=x^{2}\right)$, i.e., $\operatorname{dim} H_{+}(X, \mathbf{k})=2$.

\section{Proof of Theorem 2}

Let $\left(T\left(V_{0} \oplus V_{1} \oplus V_{2}\right), d\right) \rightarrow C_{*}(\Omega X ; \mathbf{k})$ be a free model which defines the structure of graded 3-cone on $X$. The associated $I$-adic spectral sequence satisfies then

$$
E_{-p, q}^{1}=0 \quad \text { if } 2 p>q \text { or } q>3 p .
$$

This implies

(i) $H_{1}=E_{-1,2}^{1}, H_{2}=E_{-1,3}^{1} \oplus E_{-2,4}^{2}$,

(ii) $0=d_{2}=d_{3}=\cdots$.

On the other hand, $H_{0}$ is a sub-Hopf algebra of $H(T(V), d)=\bigoplus_{i \geq 0} H_{i}$. By [5, Lemma 5], the $H_{0}$-modules $E_{-1,2}^{1}$ and $E_{-1,3}^{1}$ are free.

The differential module $\left(I / I^{2}, d_{0}\right)=\left(E_{-1,2}^{0} \oplus E_{-1,3}^{0}, d_{0}\right)$ is quasi-isomorphic to the free module $\left(E^{1}, 0\right)=\left(E_{-1,2}^{1} \oplus E_{-1,3}^{1}, 0\right)$ and then, using the Kunneth 
spectral sequence and the isomorphisms of $H_{0}$-modules $\bigotimes_{H}^{n} I / I^{2} \cong I^{n} / I^{n+1}$, we obtain an isomorphism of $H_{0}$-modules: $E^{1} \stackrel{\cong}{\rightrightarrows} T_{H_{0}}\left(E_{-1, *}^{1}\right)$. From (ii) and (i), we obtain an isomorphism of $H_{0}$-module,

$$
H_{*}(\Omega X ; \mathbf{k}) \rightarrow T_{H_{0}}\left(H_{1} \oplus M\right)
$$

where $M$ is a direct summand of the $H_{0}$-module $H_{2}$.

Then we obtain

$$
H_{1}=H_{0} \otimes U_{1}=U_{1} \otimes H_{0} \quad \text { and } \quad E_{-1,3}^{1}=H_{0} \otimes U_{2}=U_{2} \otimes H_{0}
$$

and thus the inclusion $U_{1} \oplus U_{2} \rightarrow H_{*}(\Omega X ; \mathbf{k})$ uniquely extends to an algebra homomorphism $T\left(U_{1} \oplus U_{2}\right) \rightarrow H_{*}(\Omega X ; \mathbf{k})$, and to two linear isomorphisms

$$
B \otimes T\left(U_{1} \oplus U_{2}\right) \cong H_{*}(\Omega X ; \mathbf{k}) \cong T\left(U_{1} \oplus U_{2}\right) \otimes B .
$$

\section{Proof of Theorem 3}

Let

$$
\left(T\left(V_{0} \oplus V_{1} \oplus V_{2} \oplus V_{4}\right), d\right) \rightarrow C_{*}(\Omega X ; \mathbb{Q})
$$

be a free model which defines a structure of graded 4-cone on $X$. The associated $I$-adic spectral sequence satisfies then

$$
E_{-p, q}^{1}=0 \quad \text { if } 2 p>q \text { or } q>4 p .
$$

Over the rationals, the $I$-adic spectral sequence is a spectral sequence of Hopf algebras, thus each term $E_{r, s}^{1}$ is a free $H_{0}$-module. This gives an isomorphism of $H_{0}$-modules: $E^{1} \cong T_{H_{0}}\left(E_{-1, *}^{1}\right)$. Now, the differential $d_{1}$ is completely defined by its restriction on $E_{-1,4}^{1}$ :

$$
d_{1}: E_{-1,4}^{1} \rightarrow E_{-2,4}^{2} \cong E_{-1,2}^{1} \otimes_{H_{0}} E_{-1,2}^{1} .
$$

By [13, Appendix A], $(T(V), d)$ is the enveloping algebra of a differential free bigraded Lie algebra. Hence $E^{1}=H_{0} \otimes \mathbb{U L}\left(W_{*, *}\right)$, with

$$
\begin{gathered}
W=W_{-1,2} \oplus W_{-1,3} \oplus W_{-1,4}, \\
H_{0}=\mathbf{U} L_{X}, \quad E_{1,2}^{1}=H_{0} \otimes W_{-1,2}, \\
E_{-1,3}^{1} \cong H_{0} \otimes W_{-1,3}, \quad E_{-1,4}^{1} \cong H_{0} \otimes W_{-1,4} .
\end{gathered}
$$

As, $d_{1}: W_{-1, *} \rightarrow \mathbb{L}_{-2, *}(W)$. It is clear that

$$
E_{*, *}^{2} \cong H_{0} \otimes H_{*}\left(T\left(W_{-1, *}\right), d_{1}\right)
$$

To compute $H_{*}\left(T\left(W_{-1, *}\right), d_{1}\right)$, we decompose $W_{-1, *}$ in the form

$$
W_{-1, *}=\bar{W}_{0} \oplus \bar{W}_{1}, \quad \bar{W}_{0}=W_{-1,2}, \quad \bar{W}_{1}=W_{-1,3} \oplus W_{-1,4} .
$$

The first part of Theorem 1 gives an isomorphism

$$
H_{*}\left(T\left(W_{-1, *}\right), d_{1}\right) \cong\left[T\left(W_{-1,2}\right) / d_{1}\left(W_{-1,4}\right)\right] \otimes T(U) .
$$

The preceding discussion yields thus the two following exact sequences of Hopf algebras [14]:

$$
\begin{gathered}
\mathbb{Q} \rightarrow H_{*}\left(T\left(W_{-1, *}\right), d_{1}\right) \rightarrow E^{2} \rightarrow H_{0} \rightarrow \mathbb{Q}, \\
\mathbb{Q} \rightarrow T(U) \rightarrow H_{*}\left(T\left(W_{-1, *}\right), d_{1}\right) \rightarrow T\left(L_{-1,2}\right) / d_{1}\left(L_{-1,4}\right) \rightarrow \mathbb{Q} .
\end{gathered}
$$


Moreover, as $U$ is concentrated in bidegrees $(-n-1,2 n+4)$, the differentials $d_{2}, d_{3}, \ldots$ are all trivial on $U$ by property (4) in $\S$ II. The spectral sequence collapses thus at the $E^{2}$-term.

Denote by $P$ the Lie algebra of primitive elements of $H_{*}\left(T\left(W_{-1, *}\right), d_{1}\right)$ one get a short exact sequences of Lie algebras:

$$
\begin{gathered}
0 \rightarrow P \rightarrow \pi_{*}(\Omega X) \otimes \mathbb{Q} \rightarrow L_{X} \rightarrow 0, \\
0 \rightarrow \mathbb{L}(U) \rightarrow P \rightarrow \mathbb{L}\left(W_{-1, *}\right) / d_{1}\left(\mathbb{L}\left(W_{-1,4}\right)\right) \rightarrow 0,
\end{gathered}
$$

Thus, $N=\mathbb{L}\left(W_{-1, *}\right) / d_{1}\left(\mathbb{L}\left(W_{-1,4}\right)\right)$ is the quotient of a free Lie algebra by an ideal generated by quadratic elements.

Moreover, if $X$ is a 4-cone, it is clear that the generators of $N$ are in the image of the canonical map $H_{*}\left(\Omega X_{2} ; \mathbb{Q}\right) \rightarrow H_{*}\left(\Omega X_{4} ; \mathbb{Q}\right)=H_{*}(\Omega X ; \mathbb{Q})$.

Remark finally that

$$
H_{*}\left(T\left(W_{-1, *}\right), d_{1}\right) \cong T\left(W_{-1,3}\right) *\left(H_{*}\left(T\left(W_{-1,2} \oplus W_{-1,4}\right), d_{1}\right)\right),
$$

and applying again Theorem 1 shows that

$$
H_{*}\left(T\left(W_{-1, *}\right), d_{1}\right) \cong T\left(W_{-1,3}\right) *\left(T\left(W_{-1,2}\right) / d\left(W_{-1,4}\right) \otimes T\left(U^{\prime}\right)\right),
$$

for some graded $\mathbb{Q}$-vector spaces $U^{\prime}$.

\section{REFERENCES}

1. F. Adams and P. Hilton, On the chain algebra of a loop space, Comment. Math. Helv. 30 (1955), 305-330.

2. D. Anick, Homotopy exponents for spaces of category two, Lecture Notes in Math., vol. 1370, Springer-Verlag, Berlin and New York, 1989, pp. 24-52.

3. $\frac{1}{140}$, Non commutative graded algebras and their Hilbert series, J. Algebra 78 (1982), 120140.

4. __ Hopf algebra up to homotopy, J. Amer. Math. Soc. 2 (1989), 417-453.

5. H. J. Baues, Y. Felix, and J.-C. Thomas, The Whitehead $\Gamma$-functor for chain algebras, J. Algebra 148 (1992), 123-134.

6. W. Browder, Differential Hopf algebras, Trans. Amer. Math. Soc. 107 (1963), 153-176.

7. Y. Felix, S. Halperin, C. Jacobson, C. Löwall, and J.-C. Thomas, The radical of the homotopy Lie algebra, Amer. J. Math. 110 (1988), 301-322.

8. Y. Felix, S. Halperin, J.-M. Lemaire, and J.-C. Thomas, mod p loop space homology, Invent. Math. 95 (1989), 247-262.

9. Y. Felix, S. Halperin, and J.-C. Thomas, Loop space homology of spaces of LS category one and two, Math. Ann. 287 (1990), 377-386.

10. __ Adams' cobar equivalence, Trans. Amer. Math. Soc. 329 (1992), 531-549.

11. Y. Felix and J.-C. Thomas, Sur la structure des espaces de LS catégorie deux, Illinois J. Math. 30 (1988), 574-593.

12. S. Halperin and J.-M. Lemaire, Notions of category in differential algebra, Lecture Notes in Math., vol. 1318, Springer-Verlag, Berlin and New York, 1988, pp. 138-153.

13. D. Quillen, Rational homotopy theory, Ann. of Math. (2) 90 (1969), 205-295.

14. J. Milnor and J. C. Moore, On the structure of Hopf algebras, Ann. of Math. (2) 81 (1965), 211-164.

Département de Mathématique, Université Catholique de louvain, louvain, Belgium Département de Mathématique, Université des Sciences et Techniques de Lille, Lille, France 